\title{
Effect of magnetic nanoparticles with various geometrical shapes on morphology and dielectric properties of nanodispersions of nematic liquid crystal in polymer matrix
}

\author{
P. Kopčansky ${ }^{1}$, M. Timko ${ }^{1}$, Z. Mitrova ${ }^{1}$, V. Zavisova ${ }^{1}$, M. Koneracka ${ }^{1}$, N. Tomašovičova ${ }^{1}$, L. Tomčo ${ }^{1}$, \\ O.V. Kovalchuk ${ }^{2,3}$, V.M. Bykov ${ }^{3}$, T.M. Kovalchuk ${ }^{4}$, A.I. Lad ${ }^{2}$ \\ ${ }^{1}$ Institute of Experimental Physics, Slovak Academy of Sciences, \\ 47, Watsonova str., 04001 Košice, Slovak Republic \\ ${ }^{2}$ Kyiv National University of Technologies and Design \\ 2, Nemirovich-Danchenko str., Kyiv 01011 \\ ${ }^{3}$ Institute of Physics, National Academy of Science of Ukraine, \\ 46, prospect Nauky, 03650 Kyiv, Ukraine \\ ${ }^{4}$ V. Lashkaryov Institute of Semiconductor Physics, National Academy of Sciences of Ukraine, \\ 45, prospect Nauky, 03650 Kyiv, Ukraine \\ Correspondence author e-mail: akoval@knutd.com.ua
}

\begin{abstract}
Prepared in this work are dispersions of nematic liquid crystal in polyvinyl alcohol with sizes of liquid phase inclusions less than one micrometer (nano-polymer dispersed liquid crystal (PDLC)) by changing technology of mixing the components. It was shown that, like to the case of earlier examined dispersions with the droplet sizes 6 to $10 \mu \mathrm{m}$ (micro-PDLC), the conductivity of nano-PDLC has two components: the ion one, which value does not depend on frequency, and the electron one, the value of which depends on frequency according to a power law. It was found that the ion conductivity of nano-PDLC is three orders of magnitude higher than that for micro-PDLC, and the exponent for the electron components of nano-PDLC conductivity is less than that for micro-PDLC. It was shown that the spherical and rod-like magnetic nanoparticles (MNs) influence in a different manner on the conductivity components of nano-PDLC. If for the maximum concentration of spherical MNs $2 \cdot 10^{-3} \mathrm{wt} . \%$ the ion conductivity is 2-fold increased, then at the same concentration of rod-like MNs the ion conductivity value increases by more than one order of magnitude.
\end{abstract}

Keywords: dispersion, ion conduction, electron conduction, nematic liquid crystal, polymer matrix, permittivity.

Manuscript received 11.06.13; revised version received 06.08.13; accepted for publication 19.09.13; published online 30.09.13.

\section{Introduction}

Large-scale study of nematic liquid crystal dispersion in a polymer matrix (PDLC) began from publications of the patent [1] and work [2], where it has been shown that such systems can be used to create electrooptical devices of a new type [3]. The use of these systems in display technology is limited by two significant factors, namely: a rather high Frederickzs voltage and switching time more longer than that in homogeneous liquid crystal. Currently PDLC are considered as promising materials for specific applications. As examples, one may consider curtains for windows controlled by electric field [5], fog simulators [6], UV protective sunglasses, etc. In all these devices, the mechanism of controlling the optical properties of PDLC is of fundamental importance. 
It is of interest both from the scientific and practical viewpoint to study PDLC as a visualizer of magnetic field. Due to the relatively low value of anisotropy of the diamagnetic susceptibility of liquid crystal, magneto-optical effects in PDLC are possible when sufficiently strong magnetic fields act. One can significantly enhance the effect of the magnetic field owing to introduction of magnetic nanoparticles (MNs) into PDLC. In so doing, it is important to know how MNs change the properties of PDLC.

In the work [8], we showed that, when introducing the magnetic nanoparticles of various shapes (spherical and elliptical) into PDLC, the size of the liquid crystal droplets decreases and the number of droplets with through holes increases. In this case, MNs increased the effective value of dielectric permittivity more than one order of magnitude within the frequency range $10^{-1} \ldots 10^{2} \mathrm{~Hz}$, electron and ion components of the conductivity, as well as reduced the exponent for the dependence of the electron component of conductivity on the frequency. Quantitatively, all the changes were dependent on the MN shape.

In [8], we investigated PDLC with an average size of droplets of liquid crystal 6 to $10 \mu \mathrm{m}$. In these samples, as in the case of homogenous liquid crystals, processes of nanoparticle aggregation may occur inside the LC drops. To avoid aggregation, it is of interest to obtain and investigate PDLC with much smaller sizes of LC droplets. This was the main objective of this work.

\section{Materials and methods}

The samples of PDLCs have been prepared using the following method. Liquid crystal (6CHBT) of amount $0.05 \mathrm{ml}$ was added to $5 \mathrm{ml}$ with $10 \%$ polyvinyl alcohol (PVA). The samples were sonicated with an ultrasonic oscillator (Branson model 450; $20 \mathrm{kHz}, 280 \mathrm{~W}$ ) for 1 min. A creamy white emulsion was obtained. A small drop was placed on a slide. After the water evaporation, we got a thin film. The sample thickness was $50 \mu \mathrm{m}$.

This technique was also used for preparation of the PDLC films doped with various kinds of magnetic particles. As in [8], we introduced MNs with the spherical shape and average diameter $5 \mathrm{~nm}$ as well as those with the elliptical shape and long axes 6 and $10 \mathrm{~nm}$. For each geometric shape of MNs, PDLC films with the MN concentrations $10^{-4}, 10^{-3}$ and $2 \cdot 10^{-3}$ wt.\% were prepared.

Morphology of the obtained films was investigated using the scanning electron microscope JSM-35 with the accelerating voltage $35 \mathrm{keV}$. To remove the effects of charging the surface, before the measurements a graphite film was deposited on the surface of PDLC.

Dielectric measurements were performed using the oscilloscopic method $[9,10]$ at the temperature $293 \mathrm{~K}$. We applied to the sample the alternating voltage of a triangular shape with the amplitude value $0.25 \mathrm{~V}$. The frequency range of the measuring signal was
$10^{-1} \ldots 10^{6} \mathrm{~Hz}$. The PDLC film was placed between two glass plates covered with ITO transparent layer. In order to improve the electrical contact, a small amount of 6 CHBT was deposited onto the surface of the electrodes.

\section{Results and discussion}

\subsection{The morphology of the samples}

Fig. 1 shows morphology of PDLC films with spherical MNs (a) and those with rod-like MNs (b). As follows from the data obtained in this work, in PDLC investigated the size of LC inclusions did not exceed $1 \mu \mathrm{m}$ and was about $500 \mathrm{~nm}$ on the average. This is more than an order of magnitude lower than the size of the droplets investigated in [8]. Therefore, we can consider the prepared samples as nano-PDLC. This conclusion was confirmed by the optical characteristics of the samples. If the samples studied in [8] (we name them micro-PDLC as the average size of their inclusions exceeds $1 \mu \mathrm{m}$ ) strongly scattered light in the visible spectral region and were not transparent, the nano-PDLC with the same thickness transmited light. The difference in the optical properties of nano-PDLC with PVA film was that they had an orange tint.
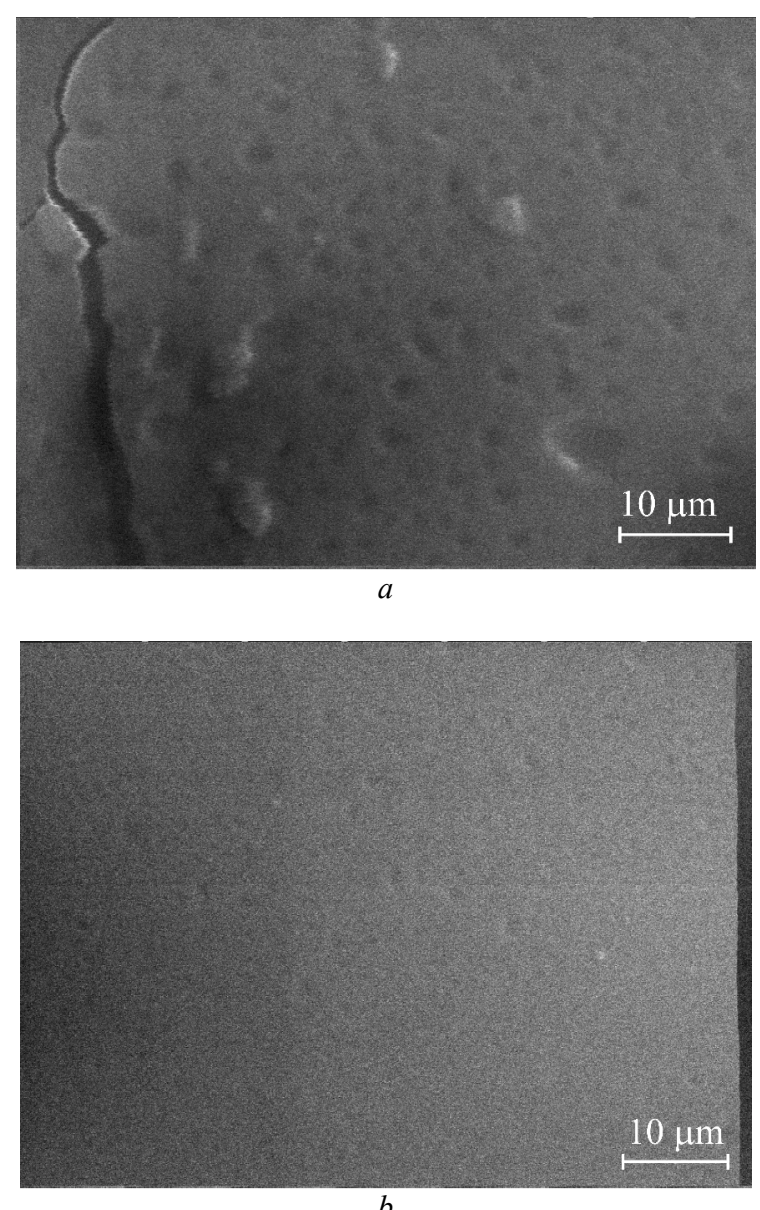

Fig. 1. The morphology of PDLC films with spherical MNs (a) and that with rod-like MNs (b). 
A comparison of Figs $1 \mathrm{a}$ and $1 \mathrm{~b}$ shows that, like to the case of micro-PDLC, morphology of nano-PDLC depends on the MN shape. Quantitative estimation was not done, as the edges of nano-inclusions were comparable with their diameter. From the data on the properties of PDLC, it is known [4] that, after separation of the phases when preparing the samples, a clear boundary between LC and polymer is not observed. There is always an intermediate layer that consists of a mixture (at the molecular level) of PVA monomers and LC molecules.

A most likely, the more accurate data on morphology for these films can be obtained when studying them with other methods, in particular AFM. These studies are planned to be held by us as a separate scientific work in future. It is important that we have prepared nano-PDLC in reality, as follows from the analysis of images obtained using a scanning electron microscope.

\subsection{The difference of dielectric spectra of nano- and micro-PDLC}

Fig. 2 shows the frequency dependences of the dielectric permittivity $\varepsilon^{\prime}$ for the films of nano-(1) and microPDLC (2). If in the case of micro-PDLC, observed is a small part of the spectrum, where the $\varepsilon^{\prime}$ value is independent of frequency $(20 \ldots 500 \mathrm{~Hz})$, in the case of nano-PDLC such part does not exist within the whole studied frequency range. The reason for the frequency dependence of $\varepsilon^{\prime}$ may be a complex process of polarization of PDLC as a heterogeneous medium. In this case, one should take into account not only the individual droplets of liquid, but also their interaction under the influence of an electric field [11]. It is important that for nano-PDLC the process of mutual polarization is pronounced significantly clearer than in the case of micro-PDLC. This may be caused by the smaller sizes of LC inclusions into nano-PDLC and smaller distances between them, respectively.

As also follows from Fig. 2, almost within the whole studied frequency range, the $\varepsilon^{\prime}$ value of nanoPDLC films is higher than that for the micro-PDLC ones. The reason for this can be not only the process of mutual polarization, but the influence of the nearelectrode processes $[12,13]$. The near-electrode processes effect more significantly at the lowest frequencies.

Fig. 3 shows the frequency dependences of the conductivity $\sigma$ for the films of nano-(1) and microPDLC (2). When analyzing these spectra, we see that each spectrum can be separated into two sections, namely: A (the frequency range of 0.5 to $200 \mathrm{~Hz}$ ) and $\mathbf{B}$ $\left(2 \cdot 10^{3} \ldots 10^{6} \mathrm{~Hz}\right)$.

In the section $\mathbf{A}$ for micro-PDLC, the $\sigma$ value does not depend on the frequency $f$. This type of conductivity, as we have proposed in [8], is caused by the ion transfer through LC. In the case of nano-PDLC also in the section $\mathbf{A}$ of the dielectric spectrum, a strong frequency dependence of $\sigma$ is absent. It may be due to the participation of another conduction mechanism beside the ion one in the nano-PDLC films.

It is important to emphasize that in the section $\mathbf{A}$ the conductivity of nano-PDLC film is three orders of magnitude higher than that for micro-PDLC one, as seen in Fig. 3. Thereof, it can be concluded that in nanoPDLC there are much more channels for ion conduction than in micro-PDLC. It may be assumed that in the nano-PDLC film, liquid crystal has the shape of ellipses or cylinders elongated in the direction perpendicular to the film surface.

The section $\mathbf{B}$ in the frequency dependence of $\sigma$, as was proposed in [8], is caused by electron conduction through polymer. In the case of PDLC, the structure of polymer is highly disordered. Therefore, this conduction occurs due to the mechanism of electron hopping from one state to another. As shown in [14], in this case the frequency dependence of the electron conductivity has the form:

$\sigma_{e}=a_{e} f^{s_{e}}$,

where $a_{e}$ is the proportionality coefficient and $s_{e}$ is the exponent.

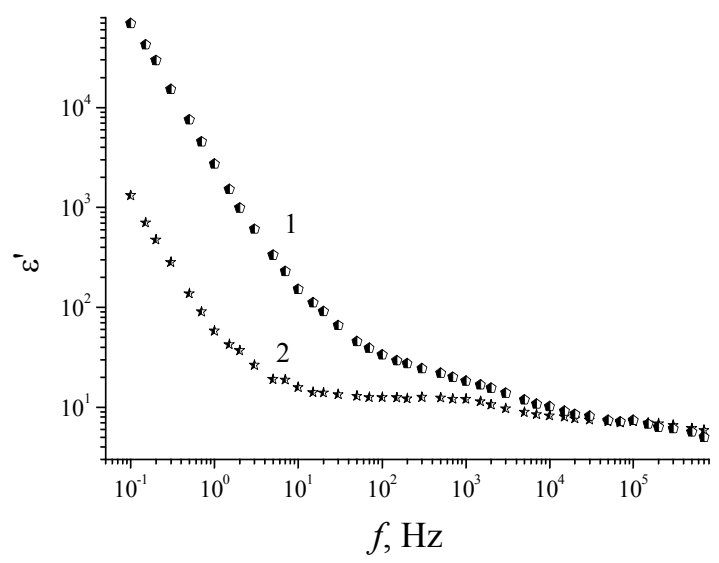

Fig. 2. Frequency dependences of the dielectric permittivity $\varepsilon^{\prime}$ for the films of nano- (1) and micro-PDLC (2).

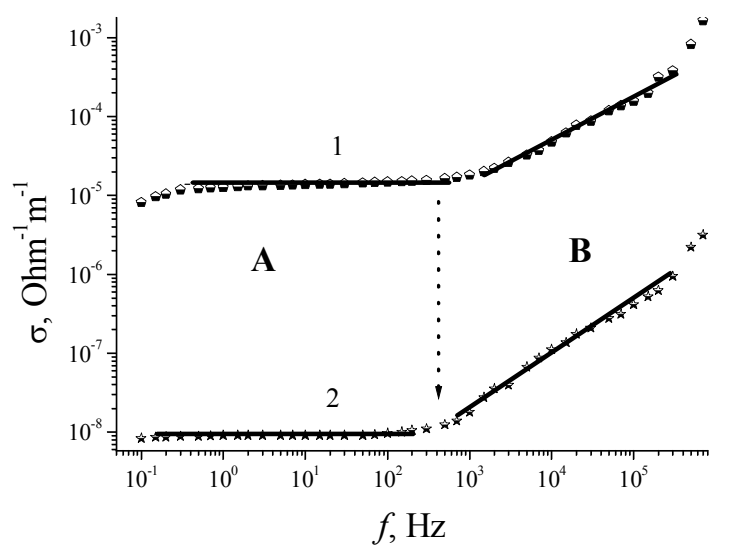

Fig. 3. Frequency dependences of the conductivity $\sigma$ for the films of nano- (1) and micro-PDLC (2). 
Table. The values of ion component of conductivity $\sigma_{i}$ and exponent of the electron component of conductivity $s_{e}$ for nanoPDLC with different concentrations of rod-like and spherical magnetic nanoparticles. Since not all the samples clearly demonstrate the part of the frequency dependence where $\sigma_{i}$ does not depend on the frequency value, the values of ion component of conductivity are listed for the frequency $100 \mathrm{~Hz}$. For comparison, the values of $\sigma_{i}$ and $s_{e}$ for micro-PDLC are shown, as well.

\begin{tabular}{|l|c|c|}
\hline Sample & $\begin{array}{c}\sigma_{i}(100 \mathrm{~Hz}), \\
\mathrm{Ohm}^{-1} \cdot \mathrm{m}^{-1}\end{array}$ & $s_{e}$ \\
\hline Micro-PDLC[8] & $9.7 \cdot 10^{-8}$ & $0.67 \pm 0.05$ \\
\hline Nano-PDLC & $1.5 \cdot 10^{-5}$ & $0.58 \pm 0.05$ \\
\hline Nano-PDLC+10 $0^{-4}$ wt.\% spherical MN & $1.4 \cdot 10^{-5}$ & $0.58 \pm 0.05$ \\
\hline Nano-PDLC+10 $0^{-3}$ wt. \% spherical MN & $1.6 \cdot 10^{-5}$ & $0.58 \pm 0.05$ \\
\hline Nano-PDLC+2.1 $10^{-3}$ wt. \% spherical MN & $3.1 \cdot 10^{-5}$ & $0.37 \pm 0.05$ \\
\hline Nano-PDLC+10 $10^{-4}$ wt. \% rod like MN & $4.3 \cdot 10^{-6}$ & $0.42 \pm 0.05$ \\
\hline Nano-PDLC+10 & $5.0 \cdot 10^{-6}$ wt. \% rod like MN & $0.42 \pm 0.05$ \\
\hline Nano-PDLC $+2 \cdot 10^{-3}$ wt. \% rod like MN & $5.1 \cdot 10^{-4}$ & $0.40 \pm 0.05$ \\
\hline
\end{tabular}

It is important to note that the $s_{e}$ values for nanoand micro-PDLC are different and equal to $0.67 \pm 0.05$ and $0.58 \pm 0.05$, respectively (Fig. 3 and Table). The very value of the electron conductivity is two orders of magnitude higher for nano-PDLC than that for microPDLC. It may be caused by more ordered structure of the polymer matrix in nano-PDLC than in micro-PDLC. Evidence of this is better mechanical properties of the nano-PDLC films.

The conductivity dependence for the whole frequency range can be represented as follows:

$$
\sigma=\sigma_{i}+a_{e} f^{s_{e}},
$$

where $\sigma_{i}$ is the ion conductivity. Here, it should be again emphasized that for nano-PDLC the ion conductivity is also weakly dependent on frequency.

\subsection{Effect of $M N$ on the dielectric properties of nano-PDLC}

Fig. 4 shows frequency dependences of the dielectric permittivity $\varepsilon^{\prime}$ for pure nano-PDLC (1) and for nanoPDLC with spherical MN impurity concentrations $10^{-4}(2), 10^{-3}(3)$, and $2 \cdot 10^{-3} \mathrm{wt} . \%$ (4). As follows from the data obtained, the most significant impact of spherical MNs is observed for the highest concentration within the frequency range $\mathbf{A}$. As it will be shown later, this may be related with a significant increase in the ion conduction component. Practically, the same changes in the $\varepsilon^{\prime}$ value are observed when introducing rodlike MNs.

The frequency dependences of conductivity $\sigma$ for pure nano-PDLC $(1)$ and for nano-PDLC with spherical MN impurity concentrations $10^{-4}(2), 10^{-3}(3)$ and $2 \cdot 10^{-3}$ wt.\% (4) are shown in Fig. 5. Similar to the case of $\varepsilon^{\prime}$, the greatest changes are observed when the MN concentration is equal to $2 \cdot 10^{-3}$ wt.\%. As follows from Fig. 5 and Table, the nanoparticles change most substantially the ion component of conduction (in the samples with the spherical MN concentration $2 \cdot 10^{-3} \mathrm{wt} . \%$ the ion component is 2 -fold increased).
Fig. 5 and Table also show that the ion conductivity of nano-PDLC depends nonmonotonically on the concentration of spherical MNs. With the spherical MN concentration $10^{-4}$ wt.\%, the nano-PDLC conductivity decreases and further with increasing the MN concentration begins to grow. We have also observed a nonmonotonic dependence of the $\sigma$ value on the $\mathrm{MN}$ concentration for mixtures of nematic liquid crystals with gold nanoparticles and nanotubes [15]. This dependence can be explained by two competing processes of conductivity changes when introducing the nanoparticles. The first process is adsorption of ions that carry charge in the liquid crystal by nanoparticles. This process leads to a decrease in conductivity. The second process is an increase of the ion conductivity due to ions that are created by the nanoparticles. Usually, the first process is revealed at low concentrations of nanoparticles, and the second one predominates at higher concentrations. We observe the decrease in the ion conductivity and then increase in it just when introducing spherical MNs into nano-PDLC (Fig. 5).

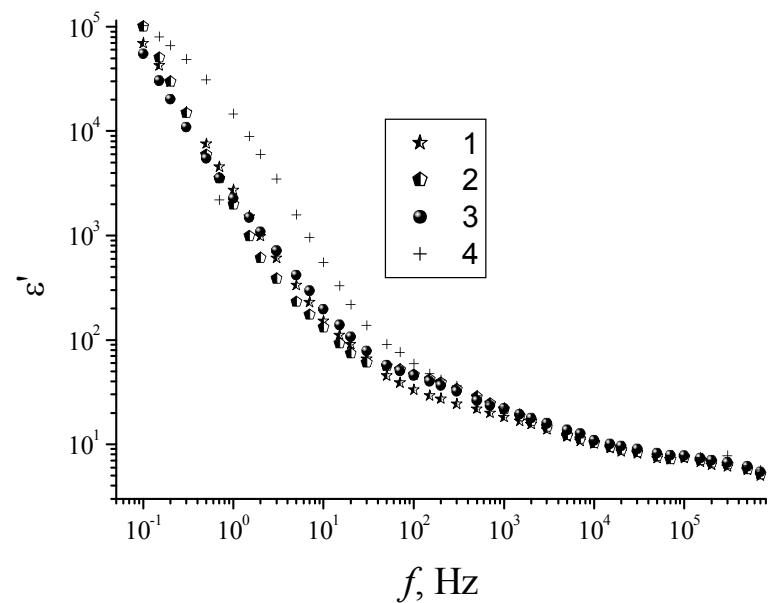

Fig. 4. Frequency dependences of the dielectric permittivity $\varepsilon^{\prime}$ for pure nano-PDLC ( 1 ) and for nano-PDLC with the spherical MN impurity concentrations $10^{-4}(2), 10^{-3}(3)$, and $2 \cdot 10^{-3}$ wt.\% (4). 


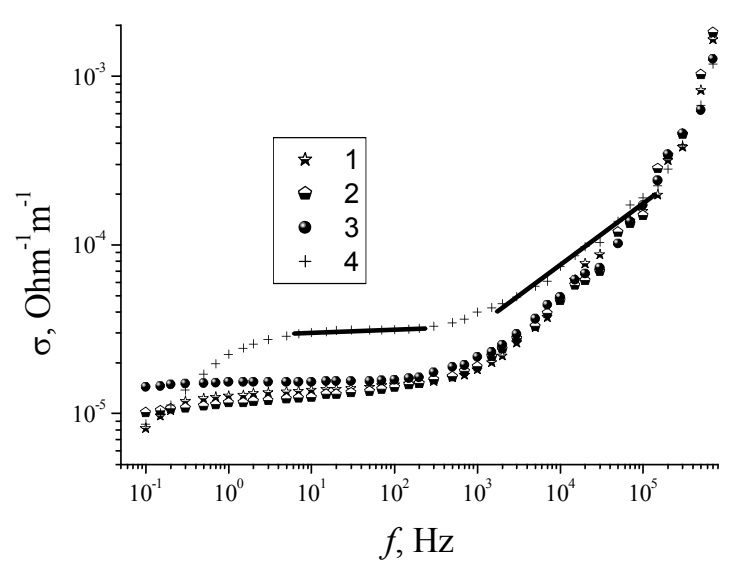

Fig. 5. Frequency dependences of the conductivity $\sigma$ for pure nano-PDLC ( 1 ) and for nano-PDLC with the spherical MN impurity concentrations $10^{-4}(2), 10^{-3}(3)$, and $2 \cdot 10^{-3}$ wt.\% (4).

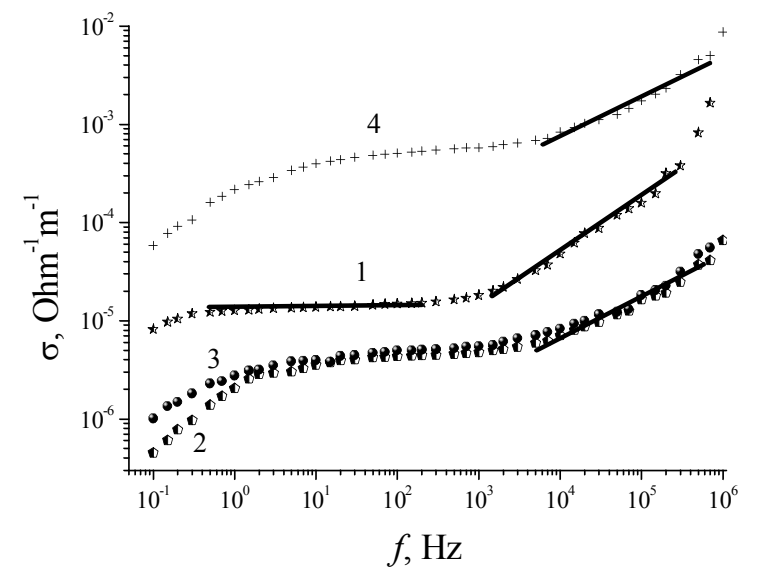

Fig. 6. Frequency dependences of the conductivity $\sigma$ for pure nano-PDLC (1) and for nano-PDLC with the rod-like MN impurity concentrations $10^{-4}(2), 10^{-3}(3)$, and $2 \cdot 10^{-3}$ wt.\% (4).

The electron conductivity of nano-PDLC changes less when introducing spherical MNs than the ion conductivity. As follows from Fig. 5, when adding spherical MNs with the concentrations $10^{-4}$ and $10^{-3} \mathrm{wt} . \%$, the conductivity of nano-PDLC does not change within the measurement errors. The most significant changes are observed for the spherical MN concentration $2 \cdot 10^{-3}$ wt.\%. This is related with a significant decrease in the $s_{e}$ value (Table). This decrease in $s_{e}$ can be explained by violation of the polymer structure, resulting in essential worsening the process of electron transfer through polymer.

Fig. 6 shows the frequency dependence of $\sigma$ for pure nano-PDLC ( 1 ) and for nano-PDLC with the rodlike MN impurity concentrations $10^{-4}(2), 10^{-3}(3)$ and $2 \cdot 10^{-3}$ wt.\% (4). As follows from this figure and Table, when introducing rod-like $\mathrm{MNs}$ the range of conductivity changes is significantly larger than that for spherical MNs. Earlier we observed different impact of spherical and rod-like MNs on the value of conductivity also for micro-PDLC [8]. However, in the case of nanoPDLC this difference is much higher. This may be related with the fact that the channels of ion conductivity in nano-PDLC have dimensions comparable with dimensions of nanoparticles.

Like to the case of spherical MNs, the dependence of ion conductivity of nano PDLC (1) on the rod-like MN concentration is nonmonotonic. When the concentration of rod-like MNs is $10^{-4} \mathrm{wt} . \%$, the ion conductivity decreases, and with the further increase in the $\mathrm{MN}$ concentration it begins to increase sharply. At the highest concentration of rod-like MNs $2 \cdot 10^{-3} \mathrm{wt} . \%$, the ion conductivity increases by more than 30 times (at the same concentration of spherical MNs it increases by only twice).

When introducing rod-like $\mathrm{MNs}$, change in the electron conductivity is also much larger than after introducing spherical MNs. From this fact, it can be concluded that the rod-like MNs influence is considerably stronger in the polymer structure than that of spherical MNs. Fig. 6 and Table show that a significant decrease in the $s_{e}$ value is already observed at the least concentration of nanoparticles $\left(10^{-4}\right.$ wt.\%). Hence, we can make the assumption that even a small concentration of rod-like MNs results in a significant change in the structure of nano-PDLC, than the same amount of spherical MNs in micro-PDLC.

\section{The main results and conclusions}

Prepared in this work are dispersions of nematic liquid crystal in polyvinyl alcohol with sizes of the liquid phase inclusions that are less than one micrometer (nanoPDLC) by changing technology of mixing the components. Using a scanning electron microscope, it has been shown that the average size of the liquid crystal inclusions is of the order of $500 \mathrm{~nm}$. Similar to the case of earlier studied micro-PDLC, this value depends on availability, concentration, and shape of the magnetic nanoparticles.

Like to micro-PDLC, the nano-PDLC permittivity depends on the frequency within the whole range under study. And in the case of nano-PDLC, the permittivity is more significant than that for micro-PDLC. It is most likely caused by more significant processes of mutual polarization due to reduction of structural elements of nano-PDLC and distances between them.

The ion conductivity of nano-PDLC is more than 3 orders of magnitude higher than that of micro-PDLC. It may be caused by significant widening the channels for ion conduction in nano-PDLC due to changes in the structure.

Increasing the electron component of conduction in nano-PDLC as compared to that in micro-PDLC is caused not only by changes in the conductivity value, but by the exponent in its dependence on the frequency. It is noteworthy that the exponent for nano-PDLC is less than that for micro-PDLC.

Introduction of magnetic nanoparticles into nanoPDLC, and in the case of micro-PDLC, leads to the 
change in the value of ion conductivity. For the same concentration, the conductivity changes are much stronger in the case of rod-like MNs than in the case of spherical MNs. The dependence of ion conductivity on the MN concentration is non-linear, which may be caused by two concentration dependent processes, namely: decrease in the number of ions in the liquid crystal (due to their adsorption on MNs) and increase in this number caused by introduction of ions together with nanoparticles.

Rod-like and spherical MNs also effect not equally on the value of the electron component of the conductivity. Rod-like MNs decrease substantially the value of the exponent for the frequency dependence of the conductivity already for the lowest concentration used in the study $\left(10^{-4}\right.$ wt.\%). At the same time, like to the case of introducing the spherical MNs into nanoPDLC, the same changes are observed at the concentration $2 \cdot 10^{-3}$ wt. $\%$. This may be related with a more significant influence of rod-like MNs than of the spherical ones on the polymer structure.

\section{References}

1. J. Fergason, Polymer encapsulated nematic liquid crystals for display and light control applications // SID Intern. Symp. Digest. Tech. Papers, No.16, p. 68 (1985).

2. J.W.Doane, N.A.Vaz, B.-G.Wu, S. Zumer, Field controlled light scattering from nematic microdroplets // Appl. Phys. Lett. 48(4), p. 269-271 (1986).

3. A.V. Kovalchuk, M.V. Kurik, O.D. Lavrentovich, Encapsulated nematic liquid crystals: A new class of display units // Zarubezhnaia radioelektronika, №5, p. 44-58 (1989), in Russian.

4. H. Stark, Physics of colloidal dispersions in nematic liquid crystals // Phys. Repts. 351(6), p. 387-474 (2001).
5. P. Kopčanský, M. Koneracká, V. Zavisova et al. Study of magnetic Fredericksz transition in ferronematics. Liquid crystals doped with fine magnetic particles // J. Phys. IV (Paris), 7, p. C1565-C1-566 (1997).

6. O. Buluy, E. Ouskova, Yu. Reznikov et al., Magnetically induced alignment of FNS // J. Magn. Magn.Mater. 252, p. 159-161(2002).

7. P. Kopčanský, N. Tomašovičová, M. Koneracká et al., Structural changes in the 6CHBT liquid crystal doped with spherical, rodlike, and chainlike magnetic particles // Phys. Rev. E, 78(1), 011702 (2008).

8. P. Kopcansky, M. Timko, Z. Mitrova, V. Zavisova, M. Koneracka, N. Tomasovicova, L. Tomco, O.P. Gornitska, O.V. Kovalchuk, V.M. Bykov, T.M. Kovalchuk, I.P. Studenyak, Morphology and dielectric properties of polymer dispersed liquid crystal with magnetic nanoparticles // Semiconductor Physics, Quantum Electronics and Optoelectronics, 13(4), p. 343-347 (2010).

9. A.J. Twarowski, A.C. Albrecht, Depletion layer in organic films: Low frequency measurements in polycrystalline tetracene // J. Chem. Phys. 20(5), p. 2255-2261(1979).

10. A.V. Koval'chuk, Low- and infralow dielectric spectroscopy liquid crystal - solid state interface. Sliding layers // Ukr. J.Phys. 41(10), p. 991-998 (1996).

11. A.V. Koval'chuk, Generation of charge carrier and formation of antisymmetric double electric layers in glycerine // J. Chem. Phys. 108(19), p. 81908194 (1998).

12. A.V. Koval'chuk, Relaxation processes and charge transport across liquid crystal - electrode interface // J. Phys.: Condens. Matter, 13(24), p. 10333 10345 (2001). 